treating patients with a proximally occluded or stenosed $\mathrm{LAD}$ or RCA. A right-sided minimally invasive CABG allows better exposure of the RCA and is recommended for those patients who require RGEA grafting. The procedure can be used with or without femorofemoral bypass. To the best of our knowledge, this is the first report of the use of bilateral minimally invasive CABG in a patient with multivessel disease. The utility of bilateral minimally invasive CABG with in situ arterial grafts has been demonstrated in the present study. The absence of a major thoracotomy may decrease patient discomfort, hospital stay, overall recovery time, and cost while providing the benefit of complete revascularization similar to that offered by standard open chest CABG. This technique is limited to revascularization of only the LAD and proximal RCA because of accessibility of the vessels. It should be emphasized that exposure of the posterior descending coronary artery for grafting is technically difficult with this approach. If the long-term results of LITA grafting to the LAD and RGEA grafting to the RCA can be accomplished with the use of this less invasive technique, a new surgical option for the management of multivessel coronary artery disease may be at hand.

\section{REFERENCES}

1. Calafiore AM, Giammarco GD, Teodori G, et al. Left anterior descending coronary artery grafting via left anterior small thoracotomy without cardiopulmonary bypass. Ann Thorac Surg 1996;61:1658-65.

2. Cosgrove DM, Sabik JF. Minimally invasive approach for aortic valve operations. Ann Thorac Surg 1996;62:596-7.

3. Subramanian VA, Sani G, Benetti FJ, Calafiore AM. Minimally invasive coronary bypass surgery: a multi-center report of preliminary clinical experience. Circulation 1995;92(Suppl): 1645.

4. Loop FD, Lytle BW, Cosgrove DM, et al. Influence of the internal mammary artery graft of 10 year survival and other cardiac events. N Engl J Med 1986;314:1-6.

5. Westaby S, Benetti FJ. Less invasive coronary surgery: consensus from the Oxford meeting. Ann Thorac Surg 1996;62: 924-31.

\title{
MIGRATION OF IMPLANTABLE CARDIOVERTER-DEFIBRILLATOR PATCH INTO THE RIGHT ATRIUM
}

\author{
Eugenio Mulero, MD, Ravindra Nath Karanam, MD, and Ryszard Krol, MD, Newark, N.J.
}

Sudden cardiac death caused by ventricular arrhythmia has been successfully aborted with implantable cardioverter-defibrillators. Because of the improvement of the design and function of the devices inserted, frequently the procedure is performed even in patients with high-risk conditions.

Transvenous systems for the most part have replaced epicardial lead systems because of the decreased morbidity and mortality. Nonetheless, on occasion technical considerations necessitate insertion of epicardial patches. Rare but serious complications can occur as in the following case with migration of the patch into one of the cardiac chambers.

Case report. A 28-year-old woman who had postpartum cardiomyopathy in 1987 and aborted sudden cardiac death underwent implantation of an epicardial defibrillator. Because of high defibrillation thresholds with a trans-

From the Department of Cardiothoracic Surgery, Department of Cardiology and Cardiac Electrophysiology, Newark Beth Israel Medical Center, Newark, N.J.

Received for publication August 30, 1996; accepted for publication Sept. 11, 1996.

J Thorac Cardiovasc Surg 1997;113:951-2

Copyright (C 1997 by Mosby-Year Book, Inc.

$0022-5223 / 97 \$ 5.00+0 \quad \mathbf{1 2 / 5 4 / 7 7 8 6 2}$ venous lead system, an epicardial system with three patches was implanted through a left subcostal approach. The patient later underwent generator change because it was approaching end of life in 1993.

In December 1995, the patient was seen because of epigastric discomfort and fever. This started an interval after a miscarriage. Pelvic inflammatory disease was ruled out. On the basis of blood cultures positive for Staphylococcus, she was given intravenous antibiotic therapy. Despite appropriate treatment, the patient continued to have febrile episodes and persistent positive blood cultures, and disseminated intravascular coagulation developed. A two-dimensional echocardiogram with a bubble test was done, which revealed a pedunculated mass in the right atrium measuring approximately 2 by $5 \mathrm{~cm}$. There was no evidence of atrial septal defect and there was no intracardiac lead suspected on echocardiography. Because of the large size and pedunculated nature of this mass, fear of massive pulmonary embolism led to the decision to remove it. In addition, because of the lack of any other source of sepsis in the presence of disseminated intravascular coagulation and persistent positive blood cultures, this mass was believed to be the source of the sepsis. While in the hospital the patient had one episode of syncopal ventricular fibrillation appropriately detected and converted to normal sinus rhythm by the defibrillator device. 


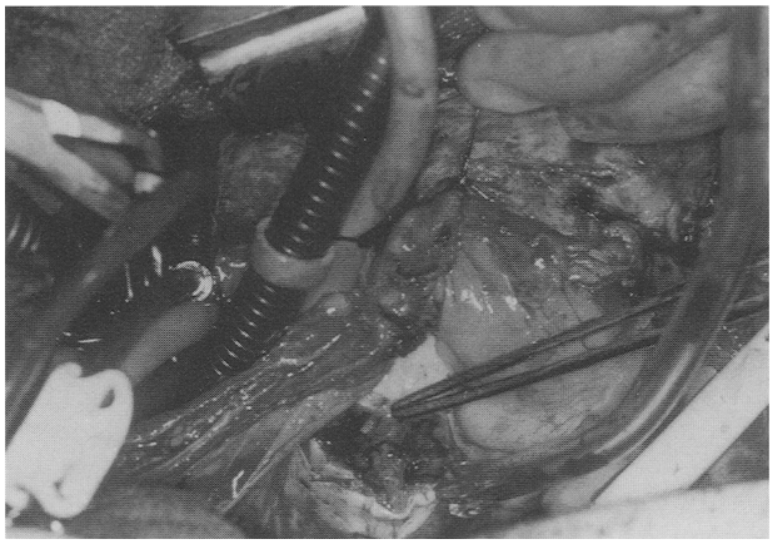

Fig. 1. Large globular thrombus (pointed to with forceps) as seen through right atriotomy.

After cardiopulmonary bypass was instituted with bicaval cannulation and moderate hypothermia, right atriotomy was done. A large globular thrombus with a pedunculated segment was evacuated and an epicardial patch was found to be inside the right atrium (Figs. 1 and 2). The patch was removed and further inspection revealed a large atrial septal defect caused by the erosion of the patch and destruction of the interatrial septum. Both the atrial septal defect and the area of migration of the anterior wall of the atrium were repaired with autogenous pericardial patches. The device and the remaining fragment of eroded lead were removed through the subcostal skin incision. The patient was successfully weaned off cardiopulmonary bypass and remained in hemodynamically stable condition but had a significant embolic cerebrovascular accident.

Discussion. Ventricular arrhythmia is one of the leading causes of sudden cardiac death. The implantable cardioverter-defibrillator has become the principal mode of therapy for a significant majority of patients with this condition. During the early evolution of these devices, epicardial lead systems, placed via thoracotomy, median sternotomy, subcostal, and subxiphoid approaches, were used commonly. Transvenous lead systems have progressively replaced the epicardial lead systems because of the efficacy and simplicity of the procedure. On occasion, technical considerations or high defibrillation thresholds, or both, necessitate alternatives such as subcutaneous patches, superior vena caval leads, and even an epicardial patch system.

New devices come with new although rare complications as described by multiple authors. ${ }^{1-3}$ Myocardial

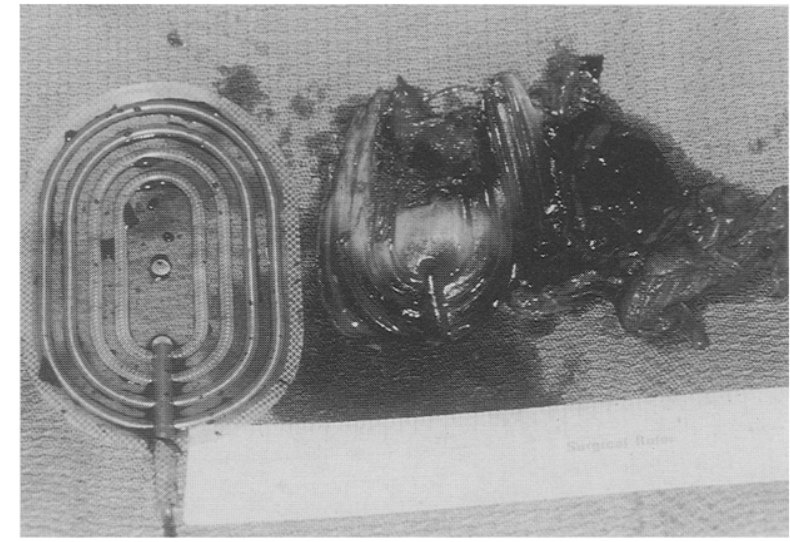

Fig. 2. Implantable cardioverter-defibrillator patch extracted from right atrium. Note the imprint over the organized thrombus.

injury with multiple, frequent discharges and erosion of the coronary arteries by the patches have been described. ${ }^{4,5}$ Crinkling of the epicardial patches not only causes failure of the system but also eventually predisposes to migration and erosion into nearby organs. ${ }^{6}$ Migration of an epicardial patch in its entirety into a cardiac chamber as seen in this patient has never been described in the medical literature.

In summary, cardiac surgeons and cardiologists must be aware that catastrophic complications with migration and erosion of epicardial patches may occur even years after implantation.

\section{REFERENCES}

1. Pfeiffer D, Jung W, Fehske W, et al. Complications of pacemaker-defibrillator devices: diagnosis and management. Am Heart J 1994;127:1073-80.

2. Grimm W, Flores B, Marchlinski FE. Complications of implantable cardioverter-defibrillator therapy: follow-up of 241 patients. Pacing Clin Electrophysiol PACE 1993;16:218-22.

3. Siclari $\mathbf{F}$, Klein $\mathbf{H}$, Troster J. Intraventricular migration of an ICD patch. Pacing Clin Electrophysiol PACE 1990;13:1356-9.

4. Feng WC, Singh AK, Chmielewski CA, Berger EE. Erosion of epicardium by intrapericardially placed ICD patch. Ann Thorac Surg 1993;56:1443-4.

5. Avitall B, Port S, Gal R, et al. Automatic implantable cardioverter/defibrillator discharges and acute myocardial injury. Circulation 1990;81:1482-7.

6. Molina JE, Benditt DG, Adler S. Crinkling of epicardial defibrillator patches: a common and serious problem. J Thorac Cardiovasc Surg 1995;110:258-64. 